\title{
Role of hybridization and on-site correlations in generating plasmons in strongly correlated $\mathrm{La}_{2} \mathrm{CuO}_{4}$
}

\author{
Muhammad Avicenna Naradipa $\odot,{ }^{1,2}$ Paolo Emilio Trevisanutto, ${ }^{1,2,3}$ Teguh Citra Asmara, ${ }^{4,2,5}$ \\ Muhammad Aziz Majidi, ${ }^{6}$ and Andrivo Rusydi $\odot^{1,2,3,4,7, *}$ \\ ${ }^{1}$ Department of Physics, National University of Singapore, Singapore 117551, Singapore \\ ${ }^{2}$ Singapore Synchrotron Light Source, National University of Singapore, Singapore 117603, Singapore \\ ${ }^{3}$ Centre for $2 D$ Advanced Materials and Graphene Research Centre, Singapore 117456, Singapore \\ ${ }^{4}$ NUSNNI-Nanocore, National University of Singapore, Singapore 117411, Singapore \\ ${ }^{5}$ Photon Science Division, Swiss Light Source, Paul Scherrer Institute, Forschungstrasse 111, CH-5232 Villigen PSI, Switzerland \\ ${ }^{6}$ Department of Physics, University of Indonesia, Depok 16424, Indonesia \\ ${ }^{7}$ NUS Graduate School for Integrative Sciences and Engineering, National University of Singapore, Singapore 117456, Singapore
}

(Received 13 June 2019; revised manuscript received 6 November 2019; accepted 22 November 2019; published 5 May 2020)

\begin{abstract}
Electronic correlation has been shown to play an important role in generating plasmons unconventionally in strongly correlated electron systems. In this work, we calculate the band structure, complex dielectric function, reflectivity, and loss function of $\mathrm{La}_{2} \mathrm{CuO}_{4}$ in its insulating and antiferromagnetic phase through first-principle calculations. We find strong evidence of unconventional plasmons that are generated by hybridizations and enhanced by on-site Coulomb correlations. Interestingly, these unconventional plasmons, predominantly located in the $\mathrm{Cu}-\mathrm{O}$ planes, are driven by hybridizations between $\mathrm{La} 5 p / \mathrm{Cu} 3 d$ and out-of-plane $\mathrm{O}_{\mathrm{z}} 2 p$. On the other hand, in-plane oxygen $\mathrm{O}_{\mathrm{xy}} 2 p$ induces conventional plasmons. This nonuniform hybridization scheme creates an anisotropic complex dielectric function and loss function, which can be influenced by conventional plasmons, unconventional plasmons, or a mixed state between both. Our result shows that the interplay of hybridizations, particularly involving oxygen, and on-site Coulomb correlations plays an important role in determining the properties of unconventional plasmons in strongly correlated $\mathrm{La}_{2} \mathrm{CuO}_{4}$.
\end{abstract}

DOI: 10.1103/PhysRevB.101.201102

Introduction. Recent theoretical studies have shown dramatic changes in plasmonic excitations under long-range Coulomb interaction and local field effect in strongly correlated electron systems [1,2]. These theoretical studies have predicted that when an effective $\left(U_{\text {eff }}\right)$ on-site Coulomb correlation is applied, conventional plasmons might become unconventional ones as plasmonic branches might form, excitations have become very local, and significant spectral weight transfer has occurred. Indeed, unconventional plasmons have been observed in transition metal oxides [3,4]. These observations open new research directions for both fundamental science and the potential to be used in optics and plasmonics, as they have multiple resonances, and are low loss and tunable [4]. This motivates us to investigate strongly correlated electron systems of layered copper oxides (cuprates) in order to explore an existence of this type of plasmons.

As a model system and the parent compound of cuprates, $\mathrm{La}_{2} \mathrm{CuO}_{4}$ has a rich and complex phase diagram, which exhibits phenomena such as structural transitions [5,6], antiferromagnetism [7], and biaxial anisotropy [8,9]. It has tetragonal crystal structure above $T_{\mathrm{C}}=533 \mathrm{~K}$ [10], similar to $\mathrm{K}_{2} \mathrm{NiF}_{4}$, where it transitions into an orthorhombic phase with slight distortions ("buckling") [6]. A magnetic transition

\footnotetext{
*phyandri@nus.edu.sg
}

has also occurred at $\approx 325 \mathrm{~K}[11,12]$, where it changes from paramagnetic to antiferromagnetic (AFM). Meanwhile, biaxial anisotropy has been confirmed through the determination of the complex dielectric function obtained from reflectivity measurements $[8,9]$. Two-dimensional correlation lengths were measured to be $1300 \mathrm{~K}$ within the $\mathrm{Cu}-\mathrm{O}$ planes $[7,11,13]$. The superconductivity phase, insulator-metal transition, and Néel temperature can be varied by doping or reducing the oxygen content $[7,11,14]$. With such rich and exotic properties, quasi-two-dimensional (quasi-2D) $\mathrm{La}_{2} \mathrm{CuO}_{4}$ is a very important material to be explored.

In this work, we show that based on first-principle calculations, unconventional plasmons are present in $\mathrm{La}_{2} \mathrm{CuO}_{4}$ and are highly sensitive to the strength of on-site correlations $U$, even without any local field effects applied. Increasing $U$ results in altering the number of excitations and therefore their intensities. Large $U$ values reduce the anisotropy and intensities of plasmonic excitations. A partial density of states (PDOS) analysis shows that the new types of plasmons are more dominant in the $\mathrm{Cu}-\mathrm{O}$ plane ( $a b$ axis) which is generated by the hybridization between $\mathrm{La} 5 p / \mathrm{Cu} 3 d$ and out-of-plane $\mathrm{O}_{\mathrm{z}} 2 p$. On the other hand, hybridization with in-plane $\mathrm{O}_{\mathrm{xy}} 2 p$ generates conventional plasmons and removes the anisotropy. The unconventional plasmons are highly sensitive to correlation effects and exist in several energy ranges, where a mixed state between the conventional and unconventional plasmons dramatically alters the material's anisotropy. 
Method. The complex dielectric function and loss function of $\mathrm{La}_{2} \mathrm{CuO}_{4}$ are calculated using spin-polarized density functional theory (DFT) calculations that use the generalized gradient approximation by Perdew-Burke-Enzerhof exchange-correlation potential from LibXC libraries and the full-potential linearized augmented plane wave method, implemented in the ELK software package [15-17]. We perform five DFT calculations for $\mathrm{La}_{2} \mathrm{CuO}_{4}$ : (1) exchange-correlation only (bare DFT), (2) antiferromagnetic (with respect to the $a$ and $b$ axes), (3) antiferromagnetic with quartered $U$ and $J$ values $(U / 4),(4)$ antiferromagnetic with halved $U$ and $J$ values $(U / 2)$, and (5) antiferromagnetic with $U$ and $J$ values equal to Czyżyk and Sawatzky $\left(U_{\text {tot }}\right)$ [18]. For all calculations we used the orthorhombic crystal structure of $\mathrm{La}_{2} \mathrm{CuO}_{4}$, where it follows the $D_{2 h}^{18} A b m a$ symmetry group. The lattice parameters $(a, b, c=5.406,5.370,13.15)$ and atom positions $\left[\mathrm{La}(0.007,0,0.362), \mathrm{Cu}(0,0,0), \mathrm{O}_{\mathrm{xy}}(0.25,0.25,0.007)\right.$, $\left.\mathrm{O}_{\mathrm{z}}(0.969,0,0.187)\right]$ are based on Grande et al. [19]. We do not account for any buckling in our structure as it is considered negligible [18]. To achieve convergence, the angular momentum cutoff for augmented plane-wave functions is $l_{\max }=272 \mathrm{eV}(10 \mathrm{Ha})$ with a $k$-point mesh of $10 \times 10 \times 10$, and furthermore, a Gaussian smearing with a smearing width of $0.01 \mathrm{Ha}(3157.8 \mathrm{~K})$.

For spin-polarized calculations, a magnetic moment in each $\mathrm{Cu}$ atom is set to be $\pm 3 \mu_{B}$, or $\approx 3.5 \times 10^{3} T$. The large magnetic field is used only for breaking spin symmetry, where this external field is reduced by $85 \%$ for each iteration. This leaves the magnetic field to be effectively infinitesimal at the end of the calculation and speed up convergence.

For DFT $+U$ calculations, we refer to Czyżyk and Sawatzky [18], whose work is similar to this study, with a focus on achieving an experimental band gap of $\mathrm{La}_{2} \mathrm{CuO}_{4}$. The $U$ and $J$ values were based on constrained local-density approximation calculation by Grant and McMahan [20], freeion optical values [21], and empirical Slater integrals [22,23]. The values we apply for this study are $U_{\mathrm{Cu}}=7.42 \mathrm{eV}, J_{\mathrm{Cu}}=$ $1.35 \mathrm{eV}, U_{\mathrm{La}}=11 \mathrm{eV}$, and $J_{\mathrm{La}}=0.68 \mathrm{eV}$. The Hubbard interaction is implemented with around-mean-field double counting method [18]. After the DFT $+U$ ground state is determined, the response function is generated from random phase approximation (RPA) [24] with the wave vector approaching zero $(q \rightarrow 0)$.

Results and discussion. After achieving the ground state energies for each DFT calculation, we calculate the linear dielectric optical response tensor using RPA. Since we are focusing on the $a, b$, and $c$ axes, the tensor elements are $\varepsilon_{11}, \varepsilon_{22}$, and $\varepsilon_{33}$. From the generated linear response function, we can calculate the complex dielectric function $\left[\varepsilon(\omega)=\varepsilon_{1}+i \varepsilon_{2}\right]$ in all three optical axes, loss function (LF) $\left(-\operatorname{Im}\left[\varepsilon^{-1}(\omega)\right]=\right.$ $\left.\left[\frac{\varepsilon_{2}}{\varepsilon_{1}^{2}+\varepsilon_{2}^{2}}\right]\right)$, and reflectivity $\left(R=\frac{(n-1)^{2}-k^{2}}{(n+1)^{2}+k^{2}}\right)$. A summary of the band gaps and magnetic moments of $\mathrm{La}_{2} \mathrm{CuO}_{4}$ is provided in Table I for $\mathrm{AFM}+U / 4, \mathrm{AFM}+U / 2$, and $\mathrm{AFM}+U_{\text {tot }}$.

We begin the analysis with a standard DFT calculation involving only exchange-correlation interaction (bare DFT). Based on band-structure calculations in Fig. 1, at this configuration $\mathrm{La}_{2} \mathrm{CuO}_{4}$ is conducting, where the states near the Fermi level are dominated by $\mathrm{Cu} 3 d$ and $\mathrm{O} 2 p$ states. The optical anisotropy is observed between the $a b$ axis and the $c$ axis, as shown in the difference between the imaginary
TABLE I. Band gap and magnetic moment of insulating $\mathrm{La}_{2} \mathrm{CuO}_{4}$. The magnetic moments are $45^{\circ}$ between the $a$ and $b$ axes, here shown as the magnitude in this direction for each $\mathrm{Cu}$ atom.

\begin{tabular}{lcc}
\hline \hline & Band gap $(\mathrm{eV})$ & Magnetic moment $\left(\mu_{B}\right)$ \\
\hline $\mathrm{AFM}+U / 4$ & 0.675 & 0.441 \\
$\mathrm{AFM}+U / 2$ & 1.287 & 0.521 \\
$\mathrm{AFM}+U_{\text {tot }}$ & 2.260 & 0.718 \\
\hline \hline
\end{tabular}

dielectric function $\left[\varepsilon_{2}(\omega)\right]$ as shown in Fig. 4. We observe conventional plasmons between 0.25 and $1 \mathrm{eV}$, created by intraband transitions from the $\mathrm{Cu} 3 d$ bands, indicated by a Drude-like peak in both $\varepsilon_{1}$ and $\varepsilon_{2}$. The metallic phase stems from DFT's underestimation of the band gap. To reveal the excitations of the insulating case, we can remove intraband contribution in the generated dielectric function calculation. In Fig. 2(d), the loss function bare DFT+Drude shows high intensity of conventional plasmons, indicated by the zero crossing at $\varepsilon_{1}$ [Fig. 2(a)]. On the other hand, the loss function of bare DFT (without any intraband contribution) is showing a peak at $1.20 \mathrm{eV}$. As there are no $\varepsilon_{1}$ crossings at this energy range, we believe this is a plasmon peak but is unconventional. In contrast to previous calculations, where local effects are used in the plane-wave DFT package [2], our calculations show that the unconventional plasmons are present even under weak correlations. This indicates that different mechanisms are at play in the 2D cuprate system compared to the quasi-1D system in niobates.

In the second set of calculations, antiferromagnetic ordering is included by doing a spin-polarized DFT calculation (AFM only). The moments for each $\mathrm{Cu}$ atom converge in the order $\pm 1 \times 10^{-3} \mu_{B}$ and no band gap is observed, which indicates a paramagnetic, metallic $\mathrm{La}_{2} \mathrm{CuO}_{4}$. Physically, we have merely assigned each electron to have spin-up and spindown states, but with no additional on-site correlations $U$ included, the calculation converges to a similar ground state to that of bare DFT. This is confirmed in the minute differences of the band structure and optical spectra (Figs. 1 and 2). With $\mathrm{DFT}+U$, we shall observe that the magnetic moments and band gap will increase by two orders of magnitude and will be closer to the experimental values as we progress.

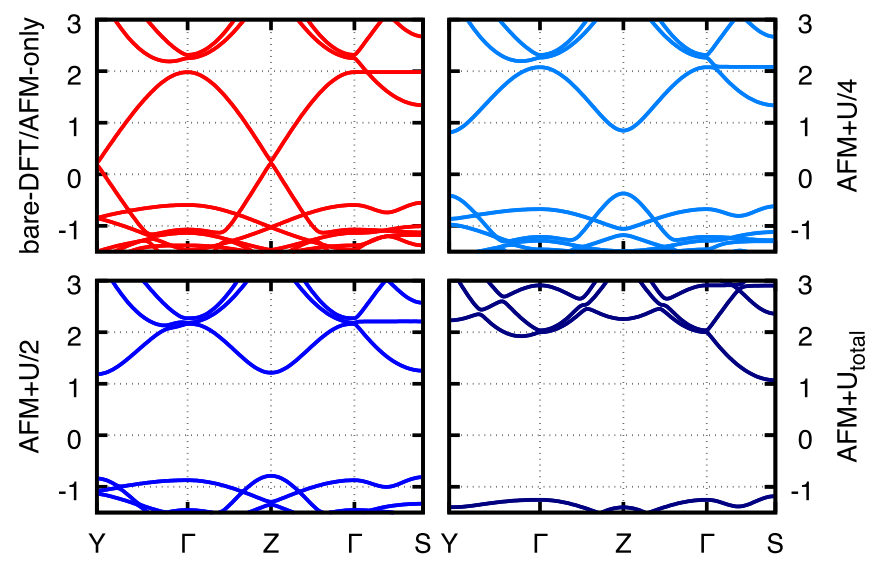

FIG. 1. Band structure of $\mathrm{La}_{2} \mathrm{CuO}_{4}$ in all calculations. Energy is in $\mathrm{eV}$ and is adjusted to $E-E_{\mathrm{Fermi}}$. 

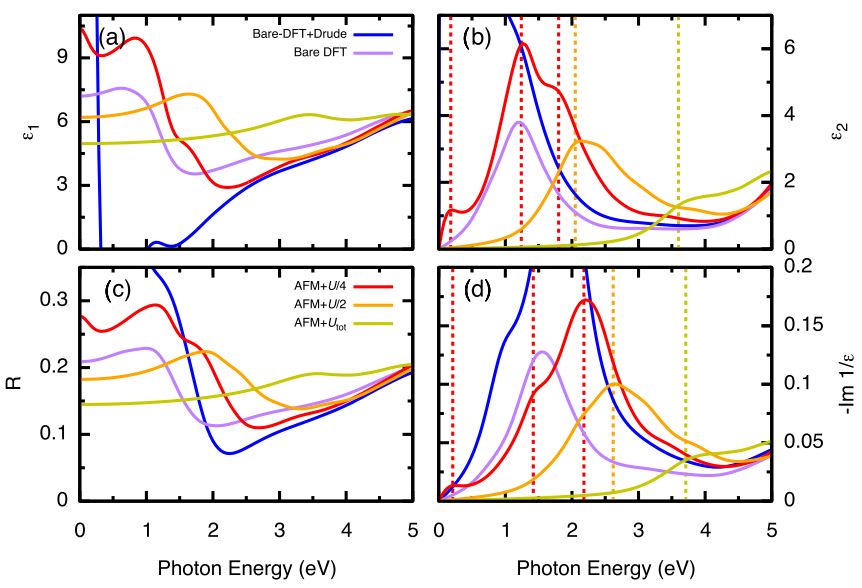

FIG. 2. Complex dielectric function, loss function (LF), and reflectivity for the $a b$ axis from 0 to $5 \mathrm{eV}$. Bare DFT and AFM-only (paramagnetic) calculations are identical.

Before applying on-site correlation $U$, we analyze the origins of the unconventional plasmons through the dominant orbitals responsible for such excitations. As shown in Fig. 3 and Supplemental Material Fig. S4 [25], we observe at $-1.20 \mathrm{eV}$, the dominant bands are $\mathrm{Cu} 3 d$ and $\mathrm{O} 2 p$ bands, which are exactly where the main excitation in bare-DFT and AFM-only calculations is located. Interestingly, we find significant differences between the band character of oxygen atoms within the $\mathrm{Cu}-\mathrm{O}$ plane $\left(\mathrm{O}_{\mathrm{xy}}\right)$ and outside of the plane $\left(\mathrm{O}_{\mathrm{z}} 2 p\right)$; they are dominant in different symmetry points energy range, where the $\mathrm{O} 2 p$ band contributions alternate between $\mathrm{O}_{\mathrm{xy}} 2 p$ and $\mathrm{O}_{\mathrm{z}} 2 p$. Furthermore, we observe between -4 and $-5 \mathrm{eV}$ (Fig. 3) that the hybridization between $\mathrm{Cu} 3 d$ and $\mathrm{O}_{\mathrm{xy}} 2 p$ does not invoke any unconventional plasmons, suggesting that it is more likely to originate from $\mathrm{O}_{\mathrm{z}} 2 p$.

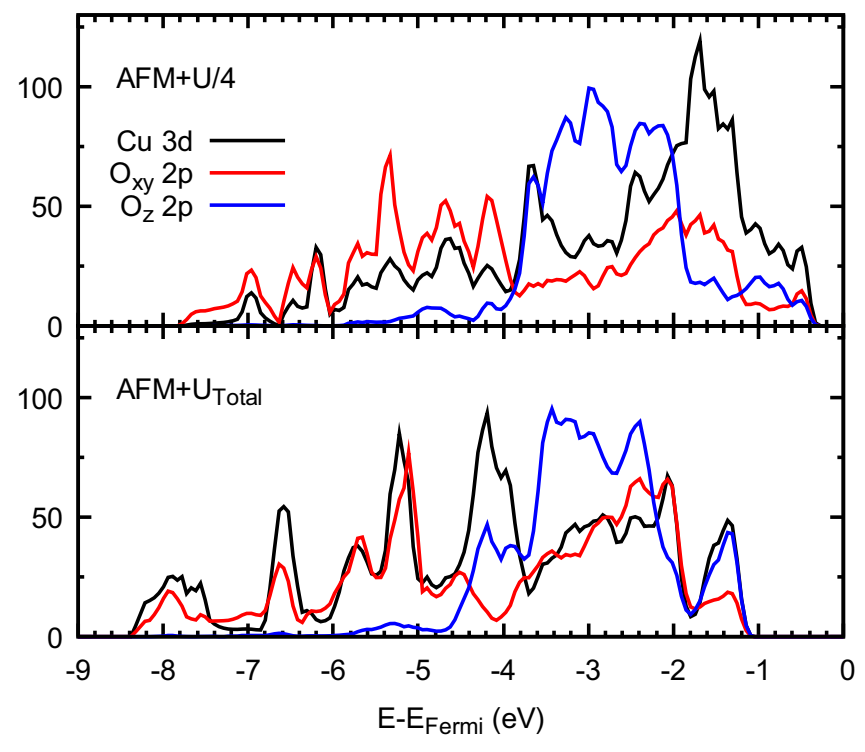

FIG. 3. Partial density of states for bare-DFT/AFM-only and $\mathrm{AFM}+U_{\text {tot }}$. Other orbital contributions are small in comparison, and thus not drawn. Energy is in $\mathrm{eV}\left(E-E_{\mathrm{Fermi}}\right)$
For the third set of calculations, we implement on-site correlation $U$ on top of the spin-polarized calculations that are used to increase the band gap. At $\mathrm{AFM}+U / 4$, the $U$ and $J$ values applied in AFM $+U / 4$ appear to be sufficient to create a meaningful gap, albeit a small one at $0.675 \mathrm{eV}$, rendering $\mathrm{La}_{2} \mathrm{CuO}_{4}$ to be insulating. The magnetic moment now converges to $\pm 0.441 \mu_{B}$, indicating that the system is an antiferromagnetic order. Surprisingly, with the addition of a small $U$, dramatic changes in the optical spectra can be seen: (1) The existence of unconventional plasmons that are not buried in a Drude-like absorption, (2) increased number of plasmonic excitations from one peak to three peaks, and (3) increased intensity of the $1.22 \mathrm{eV}$ plasmonic excitation peak. Based on the energies, the three peaks are located at 0.18 , 1.22 , and $1.8 \mathrm{eV}$. The blueshifted loss function differs by each peak, where each peak has $0.05,0.13$, and $0.4 \mathrm{eV}$ blueshift, respectively. It is clear that the peak at $0.18 \mathrm{eV}$ originates from plasmons and not interband transitions as it is below the band gap. On the other hand, the peaks at 1.22 and $1.8 \mathrm{eV}$ are located above the band gap. We analyze the band-structure plots in order to highlight the possible interband transitions that can be assigned to these peaks (see Fig. S3 in the Supplemental Material [25]). We find that all the plasmonic excitations in the loss function undergo blueshift from the absorption at $\varepsilon_{2}(\omega)$, while interband transitions have excitations that are located with the same energy in both $\varepsilon_{2}$ and LF. Other possible sources of these excitations, such as phonons, excitons, and spin-orbit interactions, are eliminated since our calculations do not include these interactions. Thus, these peaks are indeed unconventional plasmons and are sensitive to even small on-site correlation effects.

We continue our study by increasing the $U$ values. In $\mathrm{AFM}+U / 2$, the unconventional plasmons have been shifted to $2.12 \mathrm{eV}$, seen in $\varepsilon_{2}$ (Fig. 2). The band gap and moments increased to $1.287 \mathrm{eV}$ and $\pm 0.521 \mu_{B}$, respectively (Table I). This increase is expected in the treatment of DFT $+U$ or dynamical mean-field theory models, where antiferromagnetism is reinforced by the on-site correlation $U$. Interestingly, the unconventional plasmons are somewhat merged and become one broad peak, centered at $2.53 \mathrm{eV}$ with a $0.41 \mathrm{eV}$ blueshift in the LF with respect to $\varepsilon_{2}$. The shape of the peak is asymmetric, with a significantly larger FWHM compared to the plasmon peaks in $\mathrm{AFM}+U / 4$. In the loss function, the broad excitation in $\varepsilon_{2}$ undergoes a blueshift, and it is likely composed of several peaks. This indicates that the three plasmonic excitations in $\mathrm{AFM}+U / 4$ are merged due to the additional on-site correlation. Additionally, as shown from Fig. 2, the unconventional plasmon is reduced to $\approx 50 \%$, suggesting that the strongly correlated interactions initially prevalent in bare DFT and $\mathrm{AFM}+U / 4$ are competing with the applied $U$. We shall see that this intensity reduction, shift to higher energy, and merging effect are more dominant in $\mathrm{AFM}+U_{\text {tot }}$.

Finally, when applying the $U$ values taken from Czyżyk and Sawatzky [18], our calculations can replicate experimental values of the band gap and magnetic moments. It is slightly larger than previous studies at $2.26 \mathrm{eV}$ [26,27] and the magnetic moment has increased to $\pm 0.718 \mu_{B}$. Intriguingly, despite the experimental agreement in the band gap, $\mathrm{AFM}+U_{\text {tot }}$ almost completely annihilates the unconventional 


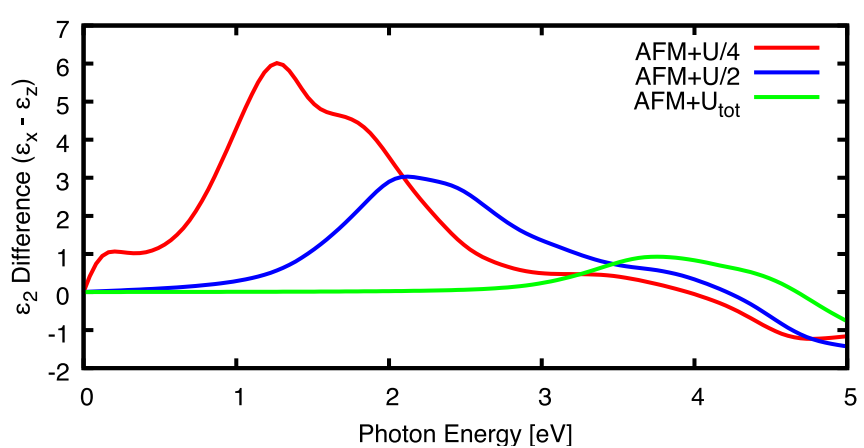

FIG. 4. Difference between imaginary dielectric function in the $a b$ axis and the $c$ axis $\left(\varepsilon_{x}-\varepsilon_{z}\right)$. For the full dielectric function of the $c$ axis, see Figs. S1 and S2 in the Supplemental Material [25].

plasmon peak. Comparisons of $\varepsilon_{2}$ in $\mathrm{AFM}+U_{\text {tot }}$ (Fig. 4) show that $\mathrm{La}_{2} \mathrm{CuO}_{4}$ is nearly isotropic up to $\approx 3.02 \mathrm{eV}$. The unconventional plasmon peak in the loss function is located at $3.68 \mathrm{eV}$ and the reflectivity minima at $\approx 4.01 \mathrm{eV}$. They have been significantly shifted to higher energies and reduced in intensity. From these observations, large on-site correlation $U$ is competing with the exchange-correlation effects already present in $\mathrm{La}_{2} \mathrm{CuO}_{4}$, resulting in an experimental band-gap value but with the expense of unconventional plasmons and anisotropic optical properties.

Examining the PDOS and band character plot (Figs. 3, S4 [25], and S5 [25]), it is clear why $\mathrm{AFM}+U_{\text {tot }}$ exhibits unusual optical properties. With the strongest $U$ value applied, the bands are compressed together along with the increased band gap. In $\mathrm{AFM}+U / 4$, the excitations are located at 0.6, 1.2, and $1.8 \mathrm{eV}$. This coincides with the $\mathrm{Cu}$ atoms hybridizing with the oxygen atoms, but the hybridization is not dominant at -0.6 and $-1.2 \mathrm{eV}$, as the oxygen hybridization is relatively low. On the other hand, for AFM $+U_{\text {tot }}, \mathrm{O}_{\mathrm{xy}} 2 p$ and $\mathrm{O}_{\mathrm{z}} 2 p$ are concentrated in the same energy level $(0$ to $-3 \mathrm{eV})$, where both have higher PDOS contribution along with $\mathrm{Cu} 3 d$ bands, indicating that they are highly hybridized. This coincides with the dielectric function becoming isotropic up to $3 \mathrm{eV}$. At $3.5 \mathrm{eV}, \mathrm{O}_{\mathrm{z}} 2 p$ is more dominant compared to $\mathrm{O}_{\mathrm{xy}} 2 p$ based on the PDOS contribution. We observe that this is the exact energy of the $3.5 \mathrm{eV}$ peak in $\varepsilon_{2}$ and the LF. Here we believe that the hybridization between $\mathrm{Cu} 3 d$ and $\mathrm{O}_{\mathrm{z}} 2 p$ might play a role in the creation of unconventional plasmons.

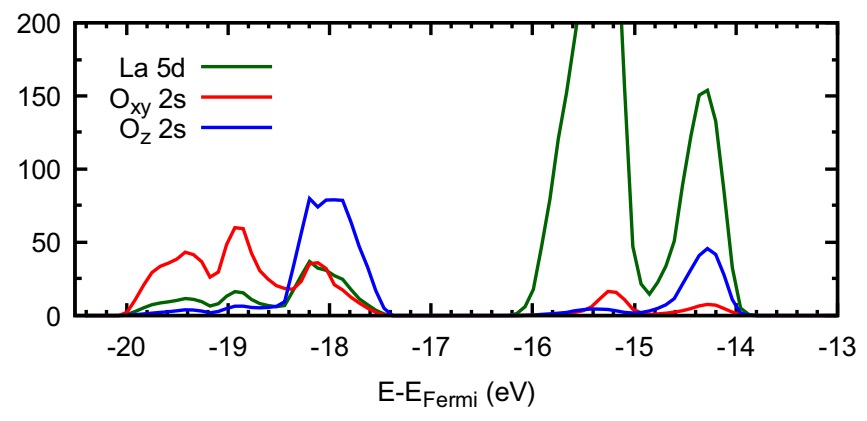

FIG. 5. Partial density of states for $\mathrm{AFM}+U_{\text {tot }}$ at $-14 \mathrm{eV}(E-$ $\left.E_{\mathrm{Fermi}}\right)$ and below.

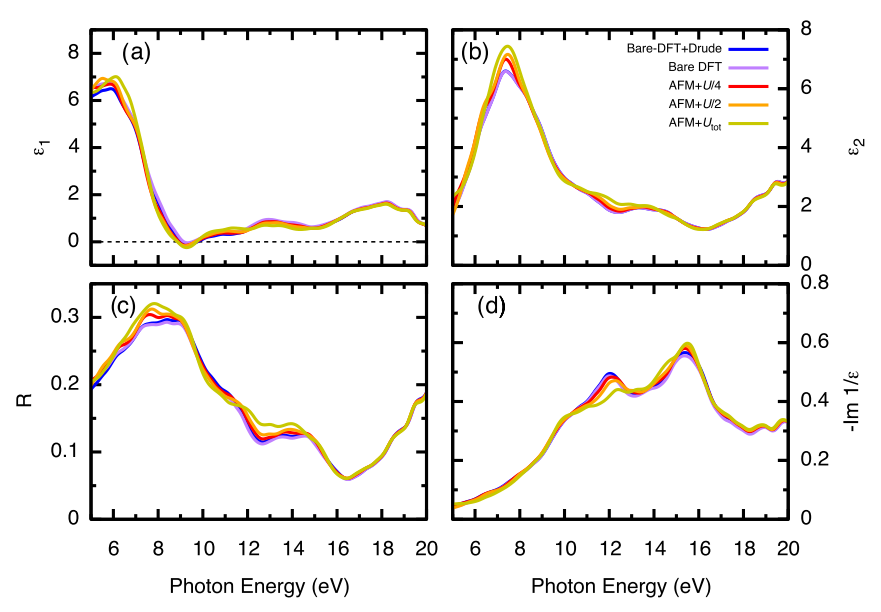

FIG. 6. Complex dielectric function, loss function (LF), and reflectivity for the $a b$ axis from 5 to $20 \mathrm{eV}$.

To further confirm our findings, we examine higher energy excitations by examining the PDOS from -5 to $-18 \mathrm{eV}$ (Figs. 5 and 6). From 5 to $10 \mathrm{eV}, \mathrm{Cu}$ atoms are hybridized with $\mathrm{O}_{\mathrm{xy}} 2 p$, but $\mathrm{O}_{\mathrm{z}} 2 p$ PDOS contribution is reduced significantly after $-4.5 \mathrm{eV}$. This region is dominated by interband transitions, since a peak is present in $\varepsilon_{2}$, but absent in the loss function. It is clear from Fig. 3 that the $\mathrm{Cu} 3 d$ hybridize with $\mathrm{O}_{\mathrm{xy}} 2 p$ at this energy range, yet produce no uncoventional plasmons. At approximately $12 \mathrm{eV}$, we observe a slight spectral weight transfer in the loss function, induced by the increase $U$. Here, we find that $\mathrm{O}_{z} 2 p$ is dominant, but is hybridized with the La $5 p$ band (Fig. 5). Its position is at approximately $-14 \mathrm{eV}$. The corresponding reduction in loss function intensity is slightly redshifted due to the conventional plasmons (indicated by $\varepsilon_{1}$ crossing zero at $8 \mathrm{eV}$ ) and interband transitions between $\mathrm{Cu} 3 d$ and $\mathrm{O}_{\mathrm{xy}} 2 p$. From these observations, $\mathrm{O}_{\mathrm{z}} 2 p$ is crucial in the creation of unconventional plasmons, to a point that its effects are seen even far below the Fermi energy. On the other hand, $\mathrm{O}_{\mathrm{xy}} 2 p$ contributes in producing conventional plasmons and interband transitions, or in other words, conforms to the band theory.

Conclusion. In conclusion, we have calculated the complex dielectric function and loss function of $\mathrm{La}_{2} \mathrm{CuO}_{4}$ in metallic and insulating phases and and have predicted the existence of unconventional plasmons in the $\mathrm{Cu}-\mathrm{O}(a b)$ plane. These excitations are sensitive to Hubbard interaction $U$, where it competes with exchange-correlation effects and hybridization between $\mathrm{La} / \mathrm{Cu}$ atoms that are predicted to be the origins of such plasmons. A better treatment of correlation effects is required to achieve both the correct band gap and optical properties for $\mathrm{La}_{2} \mathrm{CuO}_{4}$, yet it gives insight into the nature of unconventional plasmons under on-site $U$ correlation effects. Our band-structure analysis shows that the new type of plasmons is driven by hybridization between $\mathrm{La} / \mathrm{Cu}$ atoms with out-of-plane $\mathrm{O}_{\mathrm{z}} 2 p$. On the other hand, hybridization with in-plane oxygen atoms, $\mathrm{O}_{\mathrm{xy}} 2 p$, generates conventional plasmons, where a mixed state of hybridization between $\mathrm{Cu}, \mathrm{O}_{\mathrm{xy}} 2 p$, and $\mathrm{O}_{\mathrm{z}} 2 p$ removes anisotropy and all types of plasmons. These findings show that even with exchangecorrelation and a small $U$ interaction, unconventional 
plasmons may arise and are very sensitive to slight changes of the correlations at play. The unique hybridization in the $\mathrm{Cu}-\mathrm{O}$ plane may prove to be an avenue to tune exotic excitation in oxides and other strongly correlated materials.

Acknowledgments. This work is supported by Singapore National Research Foundation under its Competitive Research Funding (R-398-000-087-281), Ministry of Education of Sin- gapore AcRF Tier-2 (MOE2017-T2-1-135, MOE2018-T2-2117, and MOE2019-T2-1-163), 2015 PHC Merlion Project, and Ministry of Education of Singapore AcRF Tier-1 (R-144000-423-114, R-144-000-423-114, and R-144-000-379-114). We would like to thank the Centre for 2D Advanced Materials and Graphene Research Centre, National University of Singapore, and Lembaga Pengelola Dana Pendidikan (LPDP).
[1] E. G. C. P. van Loon, H. Hafermann, A. I. Lichtenstein, A. N. Rubtsov, and M. I. Katsnelson, Phys. Rev. Lett. 113, 246407 (2014).

[2] T. Zhu, P. E. Trevisanutto, T. C. Asmara, L. Xu, Y. P. Feng, and A. Rusydi, Phys. Rev. B 98, 235115 (2018).

[3] A. Chaudhuri, K. Rubi, T. C. Asmara, X. Chi, X. J. Yu, R. Mahendiran, and A. Rusydi, Phys. Rev. B 98, 165303 (2018).

[4] T. C. Asmara, D. Wan, Y. Zhao, M. A. Majidi, C. T. Nelson, M. C. Scott, Y. Cai, B. Yan, D. Schmidt, M. Yang et al., Nat. Commun. 8, 15271 (2017).

[5] R. Hord, G. Cordier, K. Hofmann, A. Buckow, G. Pascua, H. Luetkens, L. Alff, and B. Albert, Z. Anorg. Allg. Chem. 637, 1114 (2011).

[6] W. E. Pickett, Rev. Mod. Phys. 61, 433 (1989).

[7] A. Aharony, R. J. Birgeneau, A. Coniglio, M. A. Kastner, and H. E. Stanley, Phys. Rev. Lett. 60, 1330 (1988).

[8] R. T. Collins, Z. Schlesinger, G. V. Chandrashekhar, and M. W. Shafer, Phys. Rev. B 39, 2251 (1989).

[9] S. Uchida, T. Ido, H. Takagi, T. Arima, Y. Tokura, and S. Tajima, Phys. Rev. B 43, 7942 (1991).

[10] R. M. Fleming, B. Batlogg, R. J. Cava, and E. A. Rietman, Phys. Rev. B 35, 7191(R) (1987).

[11] G. Shirane, Y. Endoh, R. J. Birgeneau, M. A. Kastner, Y. Hidaka, M. Oda, M. Suzuki, and T. Murakami, Phys. Rev. Lett. 59, 1613 (1987).

[12] B. Keimer, A. Aharony, A. Auerbach, R. J. Birgeneau, A. Cassanho, Y. Endoh, R. W. Erwin, M. A. Kastner, and G. Shirane, Phys. Rev. B 45, 7430 (1992).

[13] V. Emery, Phys. Rev. Lett. 58, 2794 (1987).
[14] M. A. Kastner, R. J. Birgeneau, C. Y. Chen, Y. M. Chiang, D. R. Gabbe, H. P. Jenssen, T. Junk, C. J. Peters, P. J. Picone, T. Thio, T. R. Thurston, and H. L. Tuller, Phys. Rev. B 37, 111 (1988).

[15] Elk version 4.0.15, 2016.

[16] J. P. Perdew, K. Burke, and M. Ernzerhof, Phys. Rev. Lett. 77, 3865 (1996).

[17] M. A. Marques, M. J. Oliveira, and T. Burnus, Comput. Phys. Commun. 183, 2272 (2012).

[18] M. T. Czyżyk and G. A. Sawatzky, Phys. Rev. B 49, 14211 (1994).

[19] v. B. Grande, H. Müller-Buschbaum, and M. Schweizer, Z. Anorg. Allg. Chem. 428, 120 (1977).

[20] J. B. Grant and A. K. McMahan, Phys. Rev. B 46, 8440 (1992).

[21] C. Moore, Natl. Bur. Stand. (U.S.) Circ. No. 467 (U.S. GPO, Washington, D.C., 1958), Vols. 1-3 .

[22] F. M. F. de Groot, J. C. Fuggle, B. T. Thole, and G. A. Sawatzky, Phys. Rev. B 42, 5459 (1990).

[23] J. B. Mann, Atomic structure calculations. I. Hartree-fock energy results for the elements hydrogen to lawrencium, Technical Report No. LA-3690, Los Alamos Scientific Laboratory, New Mexico, 1967.

[24] P. W. Anderson, Phys. Rev. 112, 1900 (1958).

[25] See Supplemental Material at http://link.aps.org/supplemental/ 10.1103/PhysRevB.101.201102 for additional data of the dielectric properties, the band structure, and interband transitions.

[26] T. Thio, R. J. Birgeneau, A. Cassanho, and M. A. Kastner, Phys. Rev. B 42, 10800(R) (1990).

[27] S. L. Cooper, G. A. Thomas, A. J. Millis, P. E. Sulewski, J. Orenstein, D. H. Rapkine, S.-W. Cheong, and P. L. Trevor, Phys. Rev. B 42, 10785(R) (1990). 